\title{
Avaliação do volume de fluxo venoso da bomba sural por ultra-sonografia Doppler durante cinesioterapia ativa e passiva: um estudo piloto
}

\author{
Evaluation of venous flow volume of the calf muscle pump by \\ Doppler ultrasound during active and passive kinesiotherapy: \\ a pilot study
}

Carmindo Carlos Cardoso Campos ${ }^{1}$, Patrícia Cavalcanti de Albuquerque ${ }^{2}$, Ivson José da Silva Braga ${ }^{3}$

\section{Resumo}

Contexto: $\mathrm{O}$ fisioterapeuta na unidade hospitalar atua sobre os efeitos da hipoatividade ou inatividade do paciente acamado. Na prática diária, a contração do músculo da panturrilha é difundida entre os profissionais de saúde no ambiente hospitalar, principalmente nos períodos de pré e pós-operatório, como forma de diminuir a estase venosa e os riscos de trombose venosa profunda nos membros inferiores.

Objetivo: Avaliar o volume de fluxo venoso na bomba sural, através de ultra-sonografia doppler, durante cinesioterapia ativa e passiva (flexão plantar do tornozelo).

Métodos: A amostra foi constituída por 30 indivíduos escolhidos aleatoriamente e submetidos a ultra-sonografia doppler da veia poplítea direita, visando mensurar o volume de fluxo sanguíneo em quatro momentos: repouso, compressão manual da panturrilha, movimentação passiva e ativa do tornozelo em flexão plantar. $\mathrm{Na}$ análise dos resultados, utilizou-se o teste $t$, sendo utilizado um valor de $\mathrm{p} \leq 0,05$ como índice de significância estatística.

Resultados: Na amostra constituída, 16 eram do sexo feminino e 14 do sexo masculino, apresentando as seguintes médias: idade (31,57 anos), altura (1,68 m), peso $(68,25 \mathrm{~kg})$ e índice de massa corporal $(24,16)$. Na análise estatística, a flexão plantar do tornozelo realizada de forma passiva, quando comparada ao valor basal, é significante ( $\mathrm{p}$ $\leq 0,000056$ ) em relação à ativação da bomba sural, embora não tanto quanto o exercício ativo ( $\mathrm{p} \leq 0,0000016)$. Também mostrou significância a compressão manual do músculo tríceps sural em relação ao exercício passivo ( $\mathrm{p} \leq 0,000000081)$.

Conclusão: Neste estudo, a flexão plantar do tornozelo de forma ativa mostrou-se estatisticamente mais eficaz do que a passiva na ativação da bomba sural, aumentando o volume do fluxo de sangue na veia poplítea e diminuindo a estase venosa nos membros inferiores.

Palavras-chave: Fisioterapia, bomba sural, ultra-sonografia Doppler em cores.

\begin{abstract}
Background: In-hospital physical therapists work on the effects of hypoactivity or inactivity of bedridden patients. In daily practice, contraction of the calf muscle is commonly performed by health professionals in hospitals, especially in pre- and post-operative periods as a form of reducing venous stasis and risk of deep venous thrombosis in the lower limbs.
\end{abstract}

Objective: To assess venous flow volume at the calf muscle pump using color Doppler ultrasound during active and passive kinesiotherapy (ankle plantar flexion).

Methods: The sample consisted of 30 individuals randomly selected and submitted to color Doppler ultrasound of the right popliteal vein, aiming to measure blood flow volume in four periods: rest, manual calf compression, active and passive ankle movement in plantar flexion. The $t$ test was used for statistical analysis, and $\mathrm{p} \leq 0.05$ was used as an index of statistical significance.

Results: The sample consisted of 16 females and 14 males. Means were as follows: age (31.57 years), height $(1.68 \mathrm{~m})$, weight $(68.25 \mathrm{~kg})$, and body mass index (24.16). Statistical analysis showed that passive ankle plantar flexion was significant when compared to baseline $(\mathrm{p} \leq$ 0.000056 ) as to calf pump muscle activation, although not as much as active exercise $(p \leq 0.0000016)$. Manual compression of the triceps surae muscle in relation to passive exercise was also significant ( $\mathrm{p} \leq$ 0.000000081).

Conclusion: In this study, active ankle plantar flexion proved statistically more effective than passive exercise in calf muscle pump activation, increasing blood flow volume in the popliteal vein and minimizing venous stasis in the lower limbs.

Keywords: Physical therapy, calf muscle pump, color Doppler ultrasound.

1. Fisioterapeuta. Pós-graduando, Fisioterapia Hospitalar, Faculdade Redentor, Rio de Janeiro, RJ.

2. Cirurgiã vascular e ultra-sonografista, Hospital Esperança, Recife, PE.

3. Mestre em Biofísica. Professor de Biofísica e Bioestatística, Faculdade Integrada do Recife (FIR), Recife, PE, e Universidade Salgado de Oliveira (UNIVERSO), Recife, PE.

Este estudo é parte de trabalho de conclusão do Curso de Pós-Graduação em Fisioterapia Hospitalar, Faculdade Redentor, RJ.

Não foram declarados conflitos de interesse associados à publicação deste artigo.

Artigo submetido em 19.08.08, aceito em 05.11.08.

J Vasc Bras. 2008;7(4):325-332.

Copyright@ 2008 by Sociedade Brasileira de Angiologia e de Cirurgia Vascular 


\section{Introdução}

A transformação do indivíduo em paciente inclui uma série de vivências marcadas por experiências de fragmentação e perda de autonomia. A condição de restrição ao leito e distorção das relações espaciais traz para a prática médica a preocupação com os efeitos desencadeados pela imobilidade e com a qualidade do atendimento ${ }^{1}$.

A internação hospitalar gera redução da movimentação e imobilidade no leito. Pode ocasionar graves complicações nos diferentes sistemas do organismo humano, como significante diminuição na síntese protéica muscular, que já se inicia nas primeiras 9 horas de restrição ao leito ${ }^{2,3}$, gerando redução na massa muscular, diminuição da força muscular periférica, proliferação de tecido conectivo nas articulações, além de favorecer o aparecimento de encurtamentos e contraturas musculares e diminuição na massa óssea ${ }^{3-5}$. Também predispõe ao aparecimento de quadros depressivos e irritabilida$\mathrm{de}^{4,6}$, aumento da concentração sangu ínea de cortisol e exacerbação do nível de catabolismo muscular ${ }^{2,3}$. A restrição prolongada ao leito é fator de risco às vezes mais grave que a doença de base ${ }^{7}$.

Um estudo realizado em 2008 observou que os posicionamentos mais adotados por pacientes no período de internação foram o deitado e o sentado (55,5\%), enquanto que a deambulação foi 1,73\% (25 minutos por dia) ${ }^{8}$. A imobilidade prolongada no leito gera estase venosa, que é condição definida e aditiva de risco para desenvolver estados adquiridos de hipercoagulabilidade, podendo resultar em trombose venosa profunda $(\mathrm{TVP})^{9-11}$.

O combate aos efeitos negativos da imobilização pode ser alcançado através da utilização de atividade física em pacientes durante o período de internação ${ }^{12-16}$. A movimentação dos membros inferiores (MMII), principalmente na deambulação, facilita o retorno do sangue venoso neste segmento devido à ação da bomba muscular da panturrilha, também conhecida como bomba sural, reduzindo a possibilidade de estase venosa comum em pacientes restritos ao leito ${ }^{17,18}$. Um programa de exercícios no período de internação hospitalar deve incluir os MMII, a fim de melhorar a função muscular da bomba sural e auxiliar na recuperação dos problemas venosos ${ }^{18-20}$.

A patogênese da trombose venosa também está associada à presença da tríade: estase venosa, lesão endotelial da parede vascular e hipercoagulabilidade, descritas pela primeira vez em 1856 por Virchow ${ }^{21-27}$. Ocorre em $1 \%$ da população ao ano - mais de 500.000 pessoas, sendo a terceira doença cardiovascular mais comum ${ }^{25}$. É considerada de natureza multifatorial, com associação e sinergismo de fatores constitucionais e adquiri$\operatorname{dos}^{9,28}$. Os tratamentos profiláticos melhoram o fluxo sanguíneo venoso ou reduzem a coagulabilidade do san$g^{23}$. A formação do trombo pode ocorrer com facilidade no pós-operatório ao nível das cúspides valvulares das veias profundas, principalmente dos MMII. O risco de acidentes tromboembólicos persiste por um período de 3 a 4 semanas depois da cirurgia ${ }^{22}$ e aumenta consideravelmente os custos hospitalares ${ }^{9}$. A maior incidência de TVP ocorre no quarto dia do pós-operatório, e um segundo pico ocorre no $14^{\circ}$ dia $^{11,22}$.

Em revisão recente, recomendou-se que os pacientes internados fossem submetidos a protocolos de anticoagulação preventiva, conforme estratificação de risco, e que todos os pacientes fossem submetidos a medidas físicas, tais como deambulação precoce, fisioterapia, movimentação ativa ou passiva, exercícios ativos de MMII e meias elásticas de compressão pneumática intermitente. Com esses procedimentos, observou-se redução de $60 \%$ do risco relativo de $\mathrm{TEV}^{9,10,21-24,27,28}$.

O fisioterapeuta na unidade hospitalar pode atuar sobre os efeitos da hipoatividade ou inatividade do paciente acamado. Na prática diária, a contração do músculo da panturrilha e ativação da bomba sural têm sido difundidas de forma unânime entre os profissionais de saúde no ambiente hospitalar, principalmente nos períodos de pré e pós-operatório, como forma de diminuir a estase venosa nos MMII. Todos os artigos pesquisados que se referem à avaliação do fluxo sanguíneo da bomba sural são realizados com o paciente em posição ortostática, mas nenhum deles relata a avaliação deste mecanismo com o paciente deitado no leito. Entretanto, este posicionamento é o mais comum durante o período de internação hospitalar, e é neste 
Tabela 1 - Fatores de exclusão

Diabetes melito

Hipertensão arterial

Cardiopatia

Neuropatias

Artropatias

Tabagismo

Gestação

Doença arterial e/ou venosa

História pregressa de cirurgia de varizes

Portadores de malformação congênita nos MMII

Diminuição do grau de força da musculatura do tornozelo

Limitação para realizar flexão plantar do tornozelo

Alteração dos pulsos arteriais periféricos dos MMII
Queixas nos MMII*

Cansaço

Queimação

Peso e/ou edema

Cãibra

Claudicação

MMII $=$ membros inferiores

* Movimentação dos membros inferiores.

momento em que os exercícios fisioterápicos são realizados com maior freqüência.

Este estudo tem por objetivo avaliar o volume de fluxo venoso na bomba sural através de ultra-sonografia doppler durante cinesioterapia ativa e passiva (flexão plantar do tornozelo), bem como sua efetividade como forma de minimizar a estase venosa neste segmento.

\section{Materiais e métodos}

Este estudo foi desenvolvido nos meses de maio e junho de 2008. Obteve-se aprovação do Comitê de Ética e Pesquisa, conforme normas para pesquisa envolvendo seres humanos, resolução 196/96, com o número do parecer de aprovação 62/2008.
A amostra foi constituída por 30 indivíduos, de ambos os sexos, escolhidos de forma aleatória. Para ser incluído no estudo, o paciente deveria estar na faixa etária dos 21 aos 40 anos de idade, mediante assinatura do termo de consentimento livre e esclarecido. Estariam, portanto, exclusos todos os participantes que apresentassem idade inferior a 21 anos, pois não poderiam assinar legalmente o termo de consentimento, e maiores de 40 anos por apresentarem maior risco para TVP. Antes da realização do exame de ultra-sonografia Doppler, foi feita anamnese para verificar se havia algum fator de exclusão (Tabela 1).

O exame foi realizado com o paciente deitado em decúbito ventral plano, com os MMII livres de quaisquer vestimentas. Os pés ficaram para fora da mesa de 

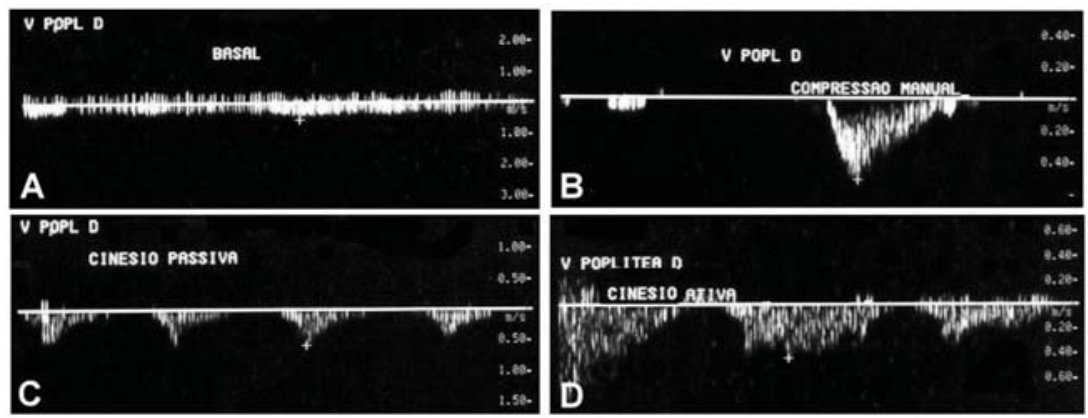

Figura 1 - Volume de fluxo sanguíneo na veia poplítea: A) fluxo basal; B) compressão manual; C) cinesioterapia passiva; D) cinesioterapia ativa

exame para permitir a mobilização do tornozelo. No primeiro instante, avaliou-se através de ultra-sonografia com Doppler colorido o calibre da veia poplítea direita; a seguir, foi mensurada a velocidade de fluxo sangu íneo em quatro momentos:

- Em repouso, sem qualquer tipo de mobilização volume de fluxo basal;

- Após manobra de compressão manual da panturrilha;

- Mediante solicitação de total relaxamento do tornozelo com mobilização passiva do tornozelo, gerando flexão plantar e hiperextensão do pé - cinesioterapia passiva;

- Após mobilização ativa do tornozelo pelo participante, com contração do tríceps sural, gerando flexão plantar e hiperextensão do pé - cinesioterapia ativa.

Entre um momento e outro, houve um período de repouso para que a bomba sural voltasse a ser preenchida por sangue venoso, possibilitando resposta fidedigna em cada momento.

O volume de fluxo sanguíneo da veia poplítea foi estudado avaliando-se calibre da veia e velocidade de fluxo na mesma, utilizando-se a fórmula: fluxo em $\mathrm{mL} / \mathrm{min}=$ área $\left(\mathrm{cm}^{2}\right) \times$ velocidade média $(\mathrm{cm} / \mathrm{s}) \times 60$. Os exames foram realizados por um único examinador. Utilizou-se o aparelho de ultra-sonografia Doppler colorido (GE LOGIC $500^{\circledR}$ ) e transdutor de 8 a $11 \mathrm{MHz}$.

Este trabalho é um estudo inferencial experimental. $\mathrm{Na}$ análise dos resultados, utilizou-se o teste $t \mathrm{e}$ o pacote computacional Statistica $^{\circledR}$ (versão 5.1), sendo utilizado um valor de $\mathrm{p} \leq 0,05$ como índice de significância estatística.

\section{Resultados}

$\mathrm{Na}$ amostra constituída por 30 pacientes de forma aleatória, 16 eram do sexo feminino e 14 do sexo masculino, apresentando as seguintes médias: idade (31,57 anos), altura (1,68 m), peso $(68,25 \mathrm{~kg})$ e índice de massa corpórea (IMC) $(24,16)$. Na anamnese, nenhum dos pacientes apresentou fatores de exclusão. Apenas três pacientes relataram praticar atividades físicas rotineiramente. Três pacientes sedentários apresentaram maior IMC na amostra (30,86/30,30/31,48), e o volume de fluxo sanguíneo mensurado durante a movimentação ativa foi inferior ao da maioria. A Figura 1 apresenta uma amostra das imagens dos volumes de fluxo venoso obtidas através de ultra-sonografia doppler em cada momento da avaliação.

Após mensuração do volume de fluxo sanguíneo venoso de todos os participantes do estudo, obtivemos os valores médios de cada momento proposto. Os resultados mostraram que a cinesioterapia (exercício) ativa apresentou valor de volume de fluxo sanguíneo bem superior ao valor basal e à cinesioterapia passiva, sendo apenas superada pela compressão manual (Tabela 2).

A Figura 2 apresenta todos os valores de volume de fluxo venoso na veia poplítea, por participante, em cada momento do estudo.

De acordo com a análise estatística, realizamos teste de significância entre os momentos do estudo e obtivemos os seguintes resultados: a flexão plantar do tornozelo realizada de forma passiva quando comparada ao 
Tabela 2 - Variação de volume de fluxo sanguíneo na veia poplítea em cada momento do estudo

\begin{tabular}{lc}
\hline Momento & Volume de fluxo (média) \\
\hline Basal & $417,28 \mathrm{~mL} / \mathrm{min}$ \\
Compressão manual & $1.444,49 \mathrm{~mL} / \mathrm{min}$ \\
Cinesioterapia passiva (mobilização) & $685,05 \mathrm{~mL} / \mathrm{min}$ \\
& $1.134,59 \mathrm{~mL} / \mathrm{min}$ \\
\hline
\end{tabular}

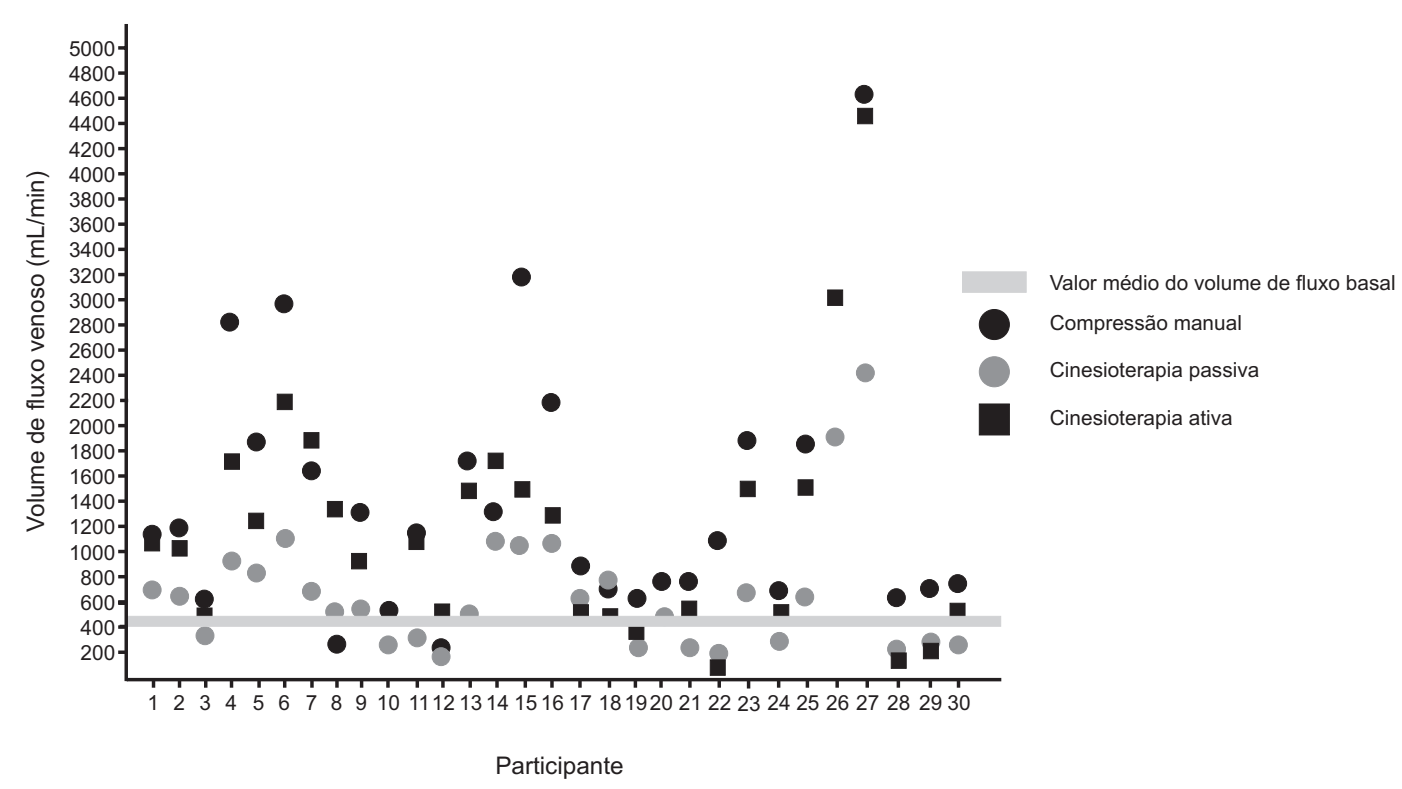

Figura 2 - Valores dos volumes de fluxo venoso $(\mathrm{mL} / \mathrm{min})$ por participante

valor basal é significante ( $\mathrm{p} \leq 0,000056)$, embora não tanto quanto o exercício ativo ( $\mathrm{p} \leq 0,0000016)$. A compressão manual do tríceps sural quando comparada à mobilização passiva apresentou grande índice de significância ( $\mathrm{p} \leq 0,000000081)$, bem como quando comparada com a mobilização ativa, apresentando $\mathrm{p} \leq 0,0008$. Também foi expressiva a significância entre exercício ativo e passivo, com $\mathrm{p} \leq 0,000006$. Portanto, a flexão plantar do tornozelo de forma ativa mostrou-se mais eficaz do que a passiva na ativação da bomba sural, com aumento do volume do fluxo de sangue na veia poplítea, minimizando a estase venosa nos MMII.

\section{Discussão}

Apesar da constatação na literatura da eficácia do funcionamento da bomba sural no retorno venoso nos MMII, não foi encontrado nenhum trabalho que confirmasse sua efetividade com o indivíduo em decúbito dorsal, posição mais adotada no período de internação hospitalar e quando são realizados os exercícios de flexão plantar do tornozelo.

Através do ultra-sonografia doppler na veia poplítea, foi avaliado e constatado que a flexão plantar do tornozelo de forma ativa gera aumento de volume de 
fluxo sanguíneo devido à eficácia da compressão muscular da bomba sural, desencadeada pelo recrutamento e contração efetiva de toda a musculatura. Com isso, há aumento do volume de fluxo venoso no membro inferior quando comparado ao valor basal e ao exercício passivo, promovendo diminuição da estase venosa neste segmento. A Figura 1D mostra o volume de fluxo na veia poplítea, quando o movimento é realizado ativamente.

Dados estatísticos deste estudo mostram que a flexão plantar do tornozelo realizada de forma passiva é eficaz quando comparada ao valor basal, embora não tanto quanto o exercício ativo. É interessante mencionar que o exercício passivo não produz contração muscular $^{18,29,30}$ e, conseqüentemente, não deveria ativar a bomba sural e aumentar o volume de fluxo sanguíneo. No momento da avaliação do volume de fluxo venoso durante o exercício passivo, foi solicitado ao paciente que deixasse o tornozelo completamente livre e sem fazer força, portanto sem contração muscular. Caso ocorresse alguma contração muscular no momento da avaliação, a mesma era desconsiderada e realizada novamente. A explicação para a ocorrência de algum tipo de contração muscular durante o exercício passivo é que, quando realizamos o movimento de flexão plantar do tornozelo passivamente em indivíduos conscientes e com reflexos motores íntegros, nos últimos graus do movimento existe uma resposta reflexa de proteção à articulação ${ }^{29,30}$. Esta resposta é desencadeada pelos receptores articulares e, neste caso, também pelos músculos dorsoflexores do tornozelo, através dos fusos musculares e dos órgãos tendinosos de Golgi ${ }^{29}$, já que este exercício provoca abertura máxima da articulação, com possível risco de lesão da mesma. Dessa forma, as fibras musculares são distendidas ao comprimento máximo, gerando um "momento de defesa" e co-contração muscular (ativação de músculos agonistas e antagonis$\operatorname{tas}^{29,30}$ ), desencadeando uma parada quase no final do arco do movimento, com ativação da bomba sural. Isto justificaria o surgimento de aumento de volume de fluxo venoso praticamente no fim do arco do movimento, quando ocorrem pequenos períodos de enchimento, que não conseguem se manter, decaindo e não sendo sucedidos de imediato por outros períodos de enchimento.
Os picos de volume de fluxo sanguíneo durante a contração passiva mostraram-se inferiores (Figura 1C) aos do exercício ativo (Figura 1D) e compressão manual (Figura 1B).

Desta forma, seria interessante realizar este estudo em indivíduos que não possuam reflexos motores, sejam temporários, como nos pacientes submetidos a bloqueio anestésico raquidiano, ou permanentes, como nos indivíduos portadores de trauma raquimedular completo. Esta eventualidade mimetizaria realmente a situação em que o exercício passivo é realizado no paciente que não possui condições de realizá-lo de forma ativa, por estar inconsciente, por apresentar fraqueza muscular acentuada ou lesão nervosa e/ou motora. Caso a hipótese de que o exercício de bomba sural, realizado de forma passiva, seja comprovadamente não eficaz para aumentar o volume de fluxo venoso, este exercício terá a finalidade apenas de preservação e manutenção da integridade articular, e não como forma de bombeamento sanguíneo no membro inferior.

Avaliamos e constatamos que a compressão manual realizada no músculo tríceps sural apresenta eficácia no aumento do volume de fluxo sanguíneo na veia poplítea, apresentando também eficácia muito elevada quando comparada ao exercício passivo. O mesmo ocorre quando comparada com o exercício ativo, mas apresentando volume de enchimento e atingindo o pico máximo de volume de fluxo de forma abrupta (Figura 1B). Isso sugere que, ao realizarmos esta manobra como, por exemplo, em uma massagem ascendente no membro inferior, devemos estar atentos à força e à pressão utilizadas, pois, como as veias têm a camada muscular menos desenvolvida que as artérias, existe o risco de lesão endotelial, facilitando o desenvolvimento de possível trombose venosa ${ }^{29}$. Deve-se prestar atenção também quando se emprega o sistema de compressão pneumática intermitente, utilizando-se como valor máximo a pressão diastólica do paciente para evitar risco de lesões ${ }^{18}$.

Os indivíduos que, na entrevista, afirmaram realizar exercícios físicos rotineiramente apresentaram maiores diâmetros da veia poplítea e picos maiores de volume de fluxo sanguíneo, pois a musculatura condicionada apresenta maior tônus, efetividade na contração e força de 
torque, demonstrando assim os efeitos benéficos da atividade física contínua sobre a musculatura esquelética e o sistema vascular e menor predisposição ao risco da trombose venosa ${ }^{30}$. Por outro lado, as pessoas sedentárias e com os maiores IMC da amostra apresentaram menor diâmetro da veia poplítea e baixo volume de fluxo sanguíneo durante a avaliação na movimentação ativa. Apesar de apresentarem uma grande perimetria na panturrilha, principalmente devido à espessa camada adiposa, a bomba sural mostrou-se descondicionada, não favorecendo de maneira esperada o aumento de volume de fluxo sanguíneo durante o exercício.

A terapia física é um fator importante na prevenção do tromboembolismo venoso, tanto no período hospitalar como após a alta do paciente, já que este risco persiste em alguns casos. Mesmo sendo um estudo piloto que utilizou uma amostra pequena de 30 indivíduos, este trabalho demonstra estatisticamente que o exercício de bomba sural (flexão plantar do tornozelo), realizado de forma ativa, com o paciente no leito, diminui a estase venosa, pois proporciona maior volume de fluxo sanguíneo no membro inferior quando comparado à forma passiva, e que o fortalecimento da musculatura da panturrilha é capaz de melhorar a hemodinâmica venosa deste segmento.

Em relação à cinesioterapia passiva, este trabalho não foi conclusivo. Nova metodologia utilizando eventualmente indivíduos com bloqueio motor temporário ou permanente seria interessante na tentativa de elucidar se a flexão plantar do tornozelo de forma passiva realmente ativa a bomba sural.

\section{Referências}

1. Toralles-Pereira ML, Sardenberg T, Mendes HWB, Oliveira RA. Communication in health: some reflections based on the perception of debridden patients in a nursery. Cienc Saude Coletiva. 2004;9:1013-22.

2. Paddon-Jones D, Wolfe RR, Ferrando AA. Amino acid supplementation for reversing bed rest and steroid myopathies. J Nutr. 2005;135:1809S-12S.

3. Andrews JR, Harrelson GL, Wilk KE. Reabilitação física das lesões esportivas. $2^{\mathrm{a}}$ ed. Rio de Janeiro: Guanabara Koogan; 2000.

4. Custaud MA, de Souza Neto EP, Abry P, et al. Orthostatic tolerance and spontaneous baroreflex sensitivity in men versus woman after 7 days of head-down bed rest. Auton Neurosci. 2002;100:66-76.
5. Kortebein P, Ferrando A, Lombeida J, Wolfe R, Evans WJ. Effect of 10 days of bed rest on skeletal muscle in healthy older adults. JAMA. 2007;297:1772-4.

6. Suesada MM, Martins MA, Carvalho CRF. Effect of short-term hospitalization on functional capacity in patients not restricted to bed. Am J Phys Med Rehab. 2007;86:455-62.

7. Krasnoff J, Painter P. The physiological consequences of bed rest and inactivity. Adv Ren Replace Ther. 1999;6:124-32.

8. Pitta F, Troosters T, Probst VS, Spruit MA, Decramer M, Gosselink R. Physical activity and hospitalization for exacerbation of COPD. Chest. 200;129:536-44.

9. Barreto SSM, Silva PM, Faccin CS, Theil AL, Nunes AH, Pinheiro CTS. Profilaxia para tromboembolia venosa em uma unidade de tratamento intensivo. J Pneumol. 2000;26:15-9.

10. Barreto SSM, Faccin CS, Silva PM, Centeno LP, Gazzana MB. Estratificação de risco e profilaxia para tromboembolia venosa em pacientes internados em hospital geral universitário. J Pneumol. 1998;24:298-303.

11. Machado FS, Martins MA, Caramelli B. Perioperatório: procedimentos clínicos. São Paulo: Sarvier; 2004.

12. Booth M. Effects of limb immobilization on skeletal muscle. J Appl Physiol. 1982;52:1113-8.

13. Artiles EM, Rodrigues M, Suárez G. El estándar de cuidados del alto riesgo de síndrome de desuso. Rev Cubana Enferm. 1997;13:54-9.

14. Biolo G, Maggi SP, Williams BD, Tipton KD, Wolfe RR. Increased rates of muscle protein turnover and amino acid transport following resistance exercise in humans. Am J Physiol. 1995;268:514-20.

15. Oliveira MSCM, Haddad ES, Koyama RCC. Síndrome da imobilização. In: Greve JMGG, Amatuzzi MM. Medicina de reabilitação aplicada à ortopedia e traumatologia. São Paulo: Roca; 1999. p. 381-98.

16. Kannus P, Jozsa L, Jarvinen TL, et al. Free mobilization and low-to-high-intensity exercise in immobilization-induced muscle atrophy. J Appl Physiol. 1998;84:1418-24.

17. Molz AB, Heyduck B, Lill H, Spanuth E, Röcker L. The effect of different exercise intensities on the fibrinolytic system. Eur J Appl Physiol Occup Physiol. 1993;67:298-304.

18. O’Sullivan SB, Schmitz TJ. Fisioterapia: avaliação e tratamento. $4^{\mathrm{a}}$ ed. São Paulo: Manole; 2004.

19. Lima RCM, Santiago L, Moura RMF, et al. Efeitos do fortalecimento muscular da panturrilha na hemodinâmica venosa e na qualidade de vida em um portador de insuficiência venosa crônica. J Vasc Bras. 2002;1:219-26.

20. Sacchi AA, Castro AA, Pitta GBB, Miranda Júnior F. Avaliação da bomba muscular da panturrilha em pacientes portadores de varizes primárias dos membros inferiores através da pletismografia a ar. J Vasc Bras. 2007;6:25-34.

21. Cassone A, Viegas AC, Sguizzatto GT, et al. Trombose venosa profunda em artroplastia total de quadril. Rev Bras Ortop. 2002;37:153-61.

22. Albuquerque HPC, Vidal PC. Trombose venosa profunda: revisão dos conceitos atuais. Rev Bras Ortop. 1996; 31:851-6. 
23. Francis CW. Clinical practice. Prophylaxis for thromboembolism in hospitalized medical patients. N Engl J Med. 2007;356:1438-45.

24. Marques LJ. Tromboembolismo pulmonar. Disponível em: http://www.fmrp.usp.br/revista/1998/vol31n2/

tromboembolismo_pulmonar.pdf

25. Fortes VB, Rollo HA, Júnior ATF, et al. Avaliação do modelo de predição clínica de Wells no diagnóstico da trombose venosa profunda dos membros inferiores. J Vasc Bras. 2007;6:7-16.

26. Cohen MP, Catalan J, Piovesan A, Chojniak R, Del Giglio A. [Prospective evaluation of clinical and Doppler ultrasound characteristics of cancer patients with suspicion of deep venous thrombosis]. Rev Assoc Med Bras. 2006;52:360-4.

27. Anaya DA, Nathens AB. Thrombosis and coagulation: deep vein thrombosis and pulmonary embolism prophylaxis. Surg Clin North Am. 2005; 85:1163-77.
28. Geerts WH, Pineo GF, Heit JA, et al. Prevention of venous thromboembolism: the Seventh ACCP Conference on Antithrombotic and Thrombolytic Therapy. Chest. 2004:126(3 Suppl):338S-400S.

29. Guyton AC, Hall JE. Tratado de fisiologia médica. $10^{\mathrm{a}}$ ed. Rio de Janeiro: Guanabara Koogan; 2002.

30. Kisner C, Colby LA. Exercícios terapêuticos: fundamentos e técnicas. $4^{\text {a }}$ ed. São Paulo: Manole; 2005.

Correspondência:

Carmindo Carlos Cardoso Campos

Rua Alcântara, 181, Coqueiral

CEP 50920-620 - Recife, PE

Tel.: (81) 9289.0018

E-mail: karlloscardoso@yahoo.com.br 